\title{
A REVIEW OF THE ACHIEVEMENTS AND POTENTIAL OF INTENSITY INTERFEROMETRY
}

R. Hanbury Brown

Astronomy Department, School of Physics, University of Sydney, Australia.

\section{ABSTRACT}

The stellar intensity interferometer at Narrabri Observatory was operated for ten years. Measurements were made of 32 single stars of spectral type 0 to $F$ and were used to establish the first completely empirical temperature scale for hot stars. The application of a high-resolution interferometer to stellar astronomy was explored: observations were made of binary, rotating and emission-line stars, limb-darkening, polarized light and of the effects of atmospheric scintillation and of Cerenkov radiation from the night sky. This programme was completed in 1973. An analysis has been made of the astronomical programme and value to stellar astronomy of a much larger and more sensitive instrument. An intensity interferometer capable of reaching stars of magnitude +7.3 with a baseline of $2 \mathrm{~km}$ has been designed and its cost has been estimated.

\section{INTRODUCTION}

Almost all our practical experience of intensity interferometry at optical wavelengths has been gained with the stellar intensity interferometer at Narrabri Observatory in New South Wales, Australia. This instrument has been described in detail elsewhere $e^{1-3}$

\section{THE INTENSITY INTERFEROMETER AT NARRABRI OBSERVATORY}

The stellar intensity interferometer was operated continuously for 10 years (1963-1973) in a programme which had three principal objectives, (a) to measure the angular diameters of a representative group of single stars: (b) to explore the astronomical applications of a high-resolution interferometer: (c) to develop the technique. 


\subsection{Measuring single stars}

The principal achievement of the programme was to measure the angular diameters of 32 single stars of spectral type 05 to F8 with an accuracy which averaged $\pm 6 \%$ (s.d) and reached $\pm 2 \%$ for the brightest stars ${ }^{4}$. These measurements were combined with photometric measurements of absolute monochromatic flux $\left(f_{\lambda}\right)$ from a star at a wavelength $\lambda$ to find the absolute emergent flux $\left(F_{\lambda}\right)$ from the surface of the star, where

$$
F_{\lambda}=4 f_{\lambda} / \theta^{2}
$$

and $\theta$ is the apparent angular diameter of the star. Measurements of $f_{\lambda}$ over the whole spectrum of the star were made, partly by satellite and partly on the ground, and were used to find the effective temperature ( $\mathrm{T}_{\mathrm{e}}$ ) where

$$
\int_{0}^{\infty} F_{\lambda} \mathrm{d} \lambda=\sigma \mathrm{T}_{\mathrm{e}}^{4}
$$

and $\sigma$ is the Stefan-Boltzmann constant.

The resulting values of $\mathrm{T}_{\mathrm{e}}$ were used to derive a temperature scale for hot stars ${ }^{5}$. It is interesting to note that, apart from minor corrections to the flux in the far ultra-violetand for the effects of 1imb-darkening, the temperature scále is based wholly on measurements and not on theoretical models. It is also interesting to note that this list of 32 single stars includes the first direct measurementsever to be made of main sequence stars.

\subsection{Astronomica1 applications}

The interferometer was designed to work only on very bright stars. For example, the signal-to-noise in a single measurement of the correlation from an unresolved star of magnitude +2 was about $3 / 1$ (r.m.s.) in one hour; as a consequence it took about $100 \mathrm{~h}$ of observation to measure the angular diameter of such a star with an uncertainty of $5 \%$. This low sensitivity seriously limited the number of applications which we could explore. Nevertheless, we did make the following experimental observations,

(i) We observed the double-line spectroscopic binary star Spica ( $\alpha$ Vir $)^{6}$. Measurements of the correlation at several baselines were made every 100 s for a total time of $84 \mathrm{~h}$ in 1966 and $115 \mathrm{~h}$ in 1970 . The results were then compared 
in a computer with a theoretical model of a binary star having 14 free parameters. Five of the parameters (epoch of periastron passage, period, eccentricity longitude and period of rotation of the line of apsides) were fixed by spectroscopic observations and the remaining parameters were fitted to the observations by the computer. In this way we found all the major parameters of the binary star, including the angle of inclination of the orbit, the masses of the two stars and the distance of the system from us. It is of considerable interest to note that this measurement of distance ( $84 \pm 4$ parsecs) is independent of interstellar extinction and is also well beyond the range of reasonably accurate trigonometrical parallaxes. Given a more sensitive interferometer this method of measuring the distance of a spectroscopic binary promises a new and powerful way of measuring distances in the Galaxy.

(ii) We observed the emission-line star $\gamma^{2}$ Velorum ${ }^{7}$.

This star is a Wolf-Rayet showing emission lines of ionized carbon; it is also a spectroscopic binary = We measured its apparent angular size in the light of ionized carbon $(465 \mathrm{~nm})$ using a narrow-band $(2.5 \mathrm{~nm})$ filter and also in the continuum at $443 \mathrm{~nm}$ using a bandwidth of $10 \mathrm{~nm}$. The results showed that the apparent angular size of the emission region in the light of lonized carbon was about five times greater than the angular size of the brighter of the two stars. This result is an interesting check on the model of a Wolf-Rayet star and it would be of considerable interest, given a more sensitive instrument, to observe other emission-1ine stars, particularly Be stars.

(iii) We measured the angular diameter of the hot star Rigel ( $\beta$ Ori) in two orthogonal planes of 1inearly polarized 1ight ${ }^{8}$. The aim of this experiment was $t$ detect the presence, and to measure the density of an extended corona and hence tc make some estimate of the rate of mass loss. A theoretical analysis shows that if the star has an ionized corona then its apparent angular size, as measured in linearly polarized light, will vary with the plane of polarization. Our observations of Rigel failed to detect any significant variation of its angular size with the plane of polarization greater than a limit of $\pm 4 \%$. Although the result, on certain simple assumptions, sets 1imits to the density of any corona of Rigel and to the mass loss, these limits are too coarse to be of much value to astrophysicists. Nevertheless, given a more sensitive instrument the technique promises an interesting way of detecting coronae around stars and of estimating the rate at which they are losing mass. 
(iv) We made observations of the effects of limb-darkening on Sirius ( $\alpha$ CMa $)^{9}$. Analysis shows that the information about the limb-darkening of a star is given by that part of the correlation-versus-baseline curve (i.e. the square of the modulus of the Fourier transform of the intensity distribution) which lies beyond the first minimum. It is in fact necessary to measure the height of the secondary maximum in the curve which, in practice, can only be done on very bright stars because it demands such a high signal/noise ratio. By observing Sirius for $203 \mathrm{~h}$ at five different baselines we were able to measure the height of the secondary maximum of the correlation curve and to show that it is consistent with a model atmosphere for the star with $\mathrm{T}_{\mathrm{e}}=10,000 \mathrm{~K}$ and $\log g=4$ where $g$ is the surface gravity. It would be of great interest to extend these measurements, with a more sensitive instrument, to supergiants and other stars with extended atmospheres.

(v) We made measurements of the rapidly rotating star Altair $(\alpha \text { Aq1 })^{3}$.

Theory tells us that rapid rotation distorts both the shape and surface brightness of a star. At the equator the radius increases and the surface brightness decreases; the reverse happens at the poles. In an attempt to observe these effects, we measured the correlation from Altair for a total of $84 \mathrm{~h}$ using north-south and east-west baselines of fixed length. As expected the correlation varied with position angle and we were able to show that this variation could be explained in terms of a model of Alta1r. The signal/noise ratio was clearly not good enough to allow any critical comparison of our results with theory There are many uncertainties about rotating stars and it would be valuable to repeat this experiment on Altair, and on some other rapidly rotating stars, with a much better signal/noise ratio.

\section{DEVELOPMENT OF THE TECHNIQUE}

The development of the actual interferometer itself, the method of observation and the many checks which we made on its reliability have been described elsewhere ${ }^{3}$. It is worth mentioning only a few major points. The major instrumental difficulty which we encountered was to develop an electronic correlator which was sufficiently free from zero-drift. We finally solved this problem and in our final design ${ }^{10}$ the zero-drift over a period of $12 \mathrm{~h}$ was less than the statistical uncertainty in the integrated output of the 
correlator due to "photon noise" alone.

A seriousconcern in the early stages of development was the possibility that Cerenkov pulses from cosmic rays in the night sky might produce a significant level of false correlation. This effect was investigated thoroughly ${ }^{11}$ and it was shown that Cerenkov pulses are not a serious limit.

Another equally important concern was to test whether or not measurements made with an intensity interferometer are significantly affected by atmospheric scintillation. We made two tests ${ }^{3}$. In the first we analyzed 236 separate runs on stars taken on 126 different nights. We compared the dispersion in the correlation observed in these runs with that to be expected due to "photon noise". There was no significant increase in this dispersion due to scintillation. In the second test we measured the correlation from the bright star Sirius ( a $\mathrm{CMa}$ ) as a function of its angle of elevation. At the same time we recorded the amplitude of scintillation in the anode currents of the phototubes. Although the scintillation increased to very large values at low angles of elevation there was no corresponding decrease in the correlatior from the star. These results confirm the important conclusion, supported by theory $^{12}$, that the results of an intensity interferometer are not significantly affected by atmospheric scintillation.

\section{A SUCCESSOR TO THE NARRABRI STELLAR INTERFEROMETER}

The stellar interferometer at Narrabri was designed to measure only very bright stars. In fact the faintest star ( $\gamma \mathrm{Crv}$ ) which was measured satisfactorily had an apparent magnitude of $+2.58(\mathrm{~V})$ Long before the observational programme was completed in 1973 we had started to think about the design of an instrument which would make it possible to extend the work to fainter stars. Historically, the major difficulties which had prevented the further development of Michelson's stellar interferometer were firstly, the mechanical problem of making an instrument of adequate size with the necessary precision and secondly, the uncertainties introduced into the measurements by atmospheric scintillation. Our work at Narrabri had demonstrated conclusively that both these difficulties can be overcome by means of an intensity interferometer and, inevitably, we looked first at the probability of modifying the existing instrument and then at the design of a completely new intensity interferometer 


\subsection{A possible astronomical programme}

We started our enquiry by estimating the performance (sensitivity, resolving power, accuracy etc.) which any new instrument must have if it is to make a significant contribution to stellar astronomy = We looked at nine possible astronomical programmes and many of our results have already been reviewed by $\mathrm{Dr}$. Davis ${ }^{13}$.

The nine programmes were,

(1) Emergent fluxes and effective temperatures of single stars.

(2) Radil of single stars.

(3) Double stars, particularly double-1ine spectroscopic binaries.

(4) Cepheid variables.

(5) Emission-line stars.

(6) Rotating stars.

(7) Limb-darkening.

(8) Interstellar absorption.

(9) Miscellaneous uses of a very large light-flux collector (e.g. Cerenkov radiation from gamma rays).

\subsection{What sensitivity is required?}

The most important programme in this list is programme (i) the measurement of the emergent fluxes and effective temperatures of single stars. As Dr. Davis ${ }^{13}$ has already pointed out the requirements for sensitivity are set by the very hot and very cool stars. Although there are about 14000 stars brighter than magnitude +7 , one must reach a limiting magnitude of $+7(\mathrm{~V})$ in order to measure a barely acceptable sample of the very hot Type 0 stars, and to measure a reasonable sample one must reach $+9.0(\mathrm{~V})$. At the other end of the spectrum the question is complicated by uncertainties about the development of photocathodes for the infra-red, nevertheless it looks as though to reach an adequate sample of very cool stars (e.g. Type M5) will require an instrument of much the same sensitivity as for the very hot stars.

As far as all the other programmes are concerned, we reached much the same conclusion. We found that a significant contribution to stellar astronomy could be made by an instrument capable of measuring stars of 
magnitude +7 , but a great deal more could be done by improving this limit to +9. As Dr Davis has shown ${ }^{13}$ this is particularly true of programme (4) on Cepheid variables. The number of Cepheids which can be measured with worthwhile accuracy by an instrument with a limiting magnitude on single stars of +7 is disappointingly smal1, only about 7. This number can be doubled by increasing the limiting magnitude to +9 and trebled by increasing it to +10 .

\subsection{What should be the length of the baseline?}

The maximum baseline which is required is set by the apparent angular diameter of the comparatively rare, and therefore distant, very hot stars. Thus to resolve a Type 05 star of magnitude +7 requires a baseline of $1.5 \mathrm{~km}$ at a wavelength of about $500 \mathrm{~nm}$; if it has a magnitude of +9 , the baseline must be nearly $4 \mathrm{~km}$. At the other end of the scale most of the cool stars, Cepheids and binary stars require much shorter $(<100 \mathrm{~m})$ baselines, and provision must be made for as wide a variety of short baselines as possible.

\subsection{How accurate must the measurements be?}

The answer to this question depends, of course, on what one hopes to do with the results. Our group in sydney is interested primarily in stars and our previous experience of applying the results from Narrabri to the problems of stellar astronomy suggests that the uncertainty in the measurements of any new interferometer must be significantly less than $\pm 5 \%$ (s.d.) To be sure, astrophysicists would 1 ike $\pm 1 \%$ but our experience suggests that such high preciston is difficult, perhaps impossible, to achieve reliably with a large and complicated instrument; there are two many minor and uncertain "systematic" effects. Nevertheless, it is of paramount importance in the development of any new stellar interferometer that it should give reliable results with an uncertainty which approaches $\pm 1 \%$. We believe that a "reasonable" target for a new instrument is $\pm 2 \%$ on bright stars, increasing to $\pm 5 \%$ for the faintest stars. 


\subsection{The specifications of a new instrument}

\subsubsection{Introduction}

As we have just seen our review of possible astronomical programmes led us to the conclusion that any successor to the interferometer at Narrabri must have sufficient sensitivity to measure stars of magnitude +7 with a baseline of about $1.5 \mathrm{~km}$ and an accuracy of at least $\pm 5 \%$. Our review also showed that it would be well worth while to extend the limiting magnitude to +9 or +10 ; this would be particularly valuable in the study of very hot stars (Type 0 ) and of Cepheid variables.

The limiting magnitude of the interferometer at Narrabri was +2.5 and to increase this to +7 (a factor of about $\times 60$ in sensitivity) was unfortunately not possible by any reasonable modification. Admittedly we might have increased it by a smaller amount, perhaps by 1 or 2 magnitudes, but the astronomical results would not, in our judgment, have justified the effort and expense. We therefore decided to design a completely new instrument.

There are two obvious ways of increasing the sensitivity, to increase the size of the flux collectors and to widen the overall electrical bandwidth of the phototubes and correlator; it is not practicable to increase the exposure times, compared with those used at Narrabri, because they were already inconveniently 1ong.

There is, however, a third possibility which has not yet been tried and that is to increase the number of independent optical channels. It can be shown ${ }^{3}$ that the signal/noise ratio of an intensity interferometer is independent of the optical bandwidth, provided that this bandwidth is large compared with the overall electrical bandwidth. It follows that by splitting the starlight into several (m) independent wavebands, each with its own phototubes and correlator, we can gain a factor of $\mathrm{m}^{\frac{1}{2}}$ in sensitivity.

The signal/noise ratio $(\mathrm{S} / \mathrm{N})$ varies with these three principal factors as,

$$
\mathrm{S} / \mathrm{N} \propto \mathrm{A}\left(\mathrm{b}_{\mathrm{v}} \mathrm{m}\right)^{\frac{1}{2}}
$$

where $A$ is the area of one of two identical flux collectors, $b_{v}$ is the overall 
electrical bandwidth and $m$ is the number of independent channels. For the interferometer at Narrabri $A=30 \mathrm{~m}^{2}, b_{v} \simeq 60 \mathrm{MHz}, \mathrm{m}=1$, and the 1imiting magnitude was $+2.5(\mathrm{~V})$.

\subsubsection{The electrical bandwidth}

How much sensitivity can we expect to gain by increasing the electrical bandwidth? At Narrabri the bandwidth was limited, not by the correlator, but by the phototubes. In recent years phototubes with greater bandwidths have been developed and it is reasonable to assume that in any future design the overall electrical bandwidth (phototubes plus correlator) could be extended to $1000 \mathrm{MHz}$. We must, however, remember that as we increase the electrical bandwidth we put more stringent requirements on the precision of the mechanical construction and movements of the interferometer. For example, if we make the bandwidth $1000 \mathrm{MHz}$ and require that any loss of correlation due to path differences or relative delays in the two sides of the interferometer should be 1 ess than $1 \%$, then we must preserve the two paths through the instrument equal to $\sim 1 \mathrm{~cm}$. Our experience at Narrabri with a system using fluxcollectors in continuous motion suggests that a mechanical precision of $1 \mathrm{~cm}$ might be achieved in a very large instrument, nevertheless, to demand an even higher precision would endanger one of the main advantages of an intensity interferometer, its freedom from the need to maintain very high mechanical precision. No doubt a higher mechanical precision, perhaps $1 \mathrm{~mm}$, could be achieved in an instrument which used fixed flux-collectors (e.g. the very large aperture synthesis radio telescope at Westerbork), 15 but it must be remembered that such high precision would not be justified because there is another, more fundamental, limit to the overall electrical bandwidth of an intensity interferometer set by the atmosphere. Atmospheric turbulence introduces random fluctuations into the time of arrival of light waves at the ground. For long baselines these fluctuations are largely uncorrelated and theory 16,17 suggests that we may write,

$$
\Delta l=0.4 \lambda\left(\mathrm{d} / \mathrm{r}_{\mathrm{o}}\right)^{5 / 6}
$$

where $\Delta l$ is the r.m.s. differential path length due to the atmosphere, $d$ is the baseline, and $r_{o}$ is the coherence length of the atmospheric 
scintillation pattern. If we take as a typical value $\mathrm{r}_{0}=10 \mathrm{~cm}$, then for a baseline of $1.5 \mathrm{~km}, \Delta l=1.5 \mathrm{~mm}$ and, for a loss of fringe visibility of $1 \%$, the electrical bandwidth would be limited to about $5000 \mathrm{MHz}$.

In summary, theory suggests that the overall electrical bandwidth of an intensity interferometer with a baseline of the order of $1 \mathrm{~km}$ would be limited by atmospheric turbulence to about $5000 \mathrm{MHz}$. On the other hand it seems likely that practical considerations such as phototubes, mechanical tolerances and expense would set a limit of about $1000 \mathrm{MHz}$.

\subsubsection{The number of separate optical channels}

How much sensitivity can we expect to gain by splitting the light into a number of independent channels? Figure 1 shows the limiting magnitude (V) of an interferometer with identical circular flux-collectors (diameter D) as a function of the number (m) of independent spectral channels. The electrical bandwidth is assumed to be $1000 \mathrm{MHz}$, the exposure time $100 \mathrm{~h}$ and the uncertainty in the final result $\pm 5 \%$.

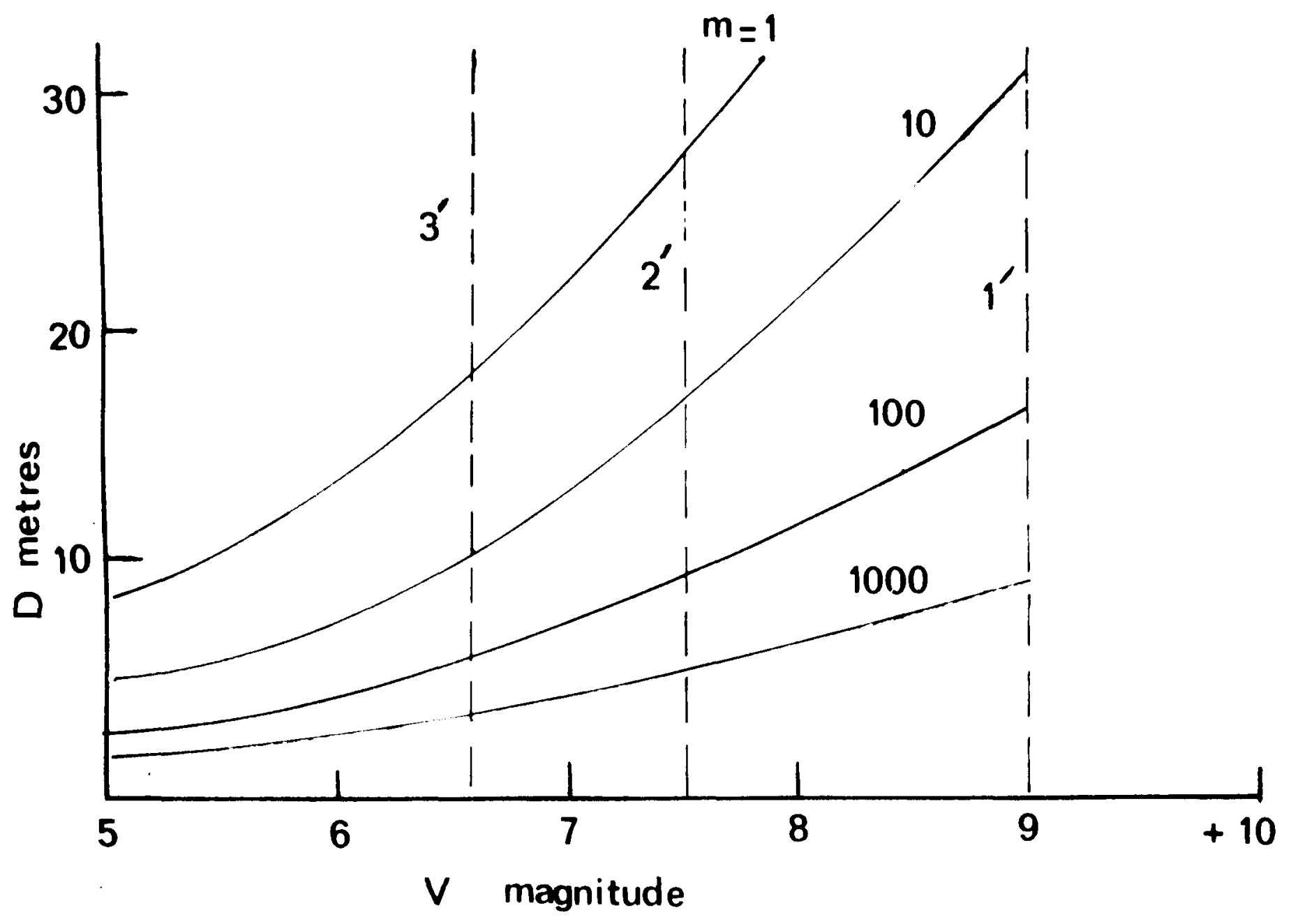

Fig. 1. The limiting magnitude (V) of an intensity interferometer with two circular flux-collectors of diameter D. The curves are shown for four different numbers (m) of optical channels. $\quad\left(\mathrm{b}_{\mathrm{v}}=1000 \mathrm{MHz}\right.$, exposure $100 \mathrm{~h}$, uncertainty in final result \pm 55 ) 
At first sight it looks simple to make a more sensitive system; all we have to do is to increase the number of spectral channels For example, Figure 1 suggests that we could reach magnitude t9 with a $10 \mathrm{~m}$ flux-collector and 1000 spectral channels or, if we prefer, with a $30 \mathrm{~m}$ flux-collector and 10 channe1s. However, a closer look at this problem shows that the use of a very large number of optical channels is not practical. Thus, if we use a diffraction grating or prism to disperse the light, the minimum separation between adjacent channels is set by angular dispersion in the collimated beam which, in turn, is governed by the optical quality of the flux-collector, the effects of atmospheric scintillation and the size of the secondary optical system. For example, if we assume that our $10 \mathrm{~m}$ reflector is perfect and that the diameter of the secondary optical system is $15 \mathrm{~cm}$, then the effects of atmospheric scintillation alone would limit the minimum spacing between adjacent spectral channels to the order of $1 \mathrm{~nm}$; it would therefore be impossible to use 1000 channels and hence we would not reach a limiting magnitude of +9 . A detailed analysis shows that it is not practicable to reach the sensitivity which we need by using comparatively small flux-collectors and large numbers of optical channels; such systems would be excessively complicated and expensive. The only practicable alternative is to use very large and relatively crude flux-collectors of "search-1ight" or "solar furnace" quality. Unfortunately the light from such collectors is poorly collimated and we cannot use conventional methods, such as prisms or diffraction gratings, to disperse it into independent spectral channels. The only attractive solution which we have found so far is to use "cascaded" interference filters. We have designed an optical system for a very large flux-collector in which the light is separated into two orthogonal polarizations and then passed through a sequence of interference filters each with its own bandpass, phototubes and correlator. Again, the maximum number of channels, and hence the overall gain in sensitivity, is limited by the angular dispersion in the collimated beam. Taking the angular size of the image of a star (in the plane of the sky) to be 2 mins of arc and the diameter of the flux-collector and of the filters to be $13 \mathrm{~m}$ and $15 \mathrm{~cm}$ respectively, we found that the maximum useful number of separate channels is limited to 10 and that the overall gain in sensitivity would only be $\times 2.9$ as compared with $\times 3.2$ for an ideal system with no losses. 


\subsubsection{The size of the flux-collectors.}

Finally how large can we make the flux-collectors? Figure 1 shows that, if we use two identical circular flux-collectors and 10 independent optical channels $(m=10)$, then to reach a limiting magnitude of +7 the diameter of our flux-collector must be at least $12 \mathrm{~m}$. If we want to reach +9 they must have a diameter of no less than $30 \mathrm{~m}$ ! Apart from the obvious question of cost there are other constraints. One major constraint is that their optical quality must be sufficiently high to restrict the light from the night sky to a small fraction (say < 10\%) of the light received from the faintest star to be measured. Thus, if we wish to measure stars of magnitude +7 at a wavelength of about $500 \mathrm{~nm}$, then the field of view of our flux-collectors cannot exceed about $2 \frac{1}{2}$ mins of arc. The corresponding magnitudes for three other fields of view are shown in Figure 1 by the broken vertical lines. They show that although we can reach fainter stars by building larger reflectors, the optical quality and therefore the cost of these reflectors increases rapidly; for example, if we aim to reach +9 by building a $30 \mathrm{~m}$ flux-collector $(\mathrm{m}=10)$ then the field of view must not exceed 1 min of arc which would make it extremely expensive. Apart from cost, there are constraints on the size of individual flux-collectors. For example, they must not be so large that they partially resolve some of the single or double stars which we wish to measure. Another constraint is that, for a given angular aberration in the collimated beam the diameter of the secondary optical system (interference filters etc.) increases directly with the diameter of the flux-collector and, beyond a certain limit, it becomes too difficult and expensive to design.

An obvious way out of these difficulties is to use a number of smaller flux-collectors. As we shall see in the next section, our proposed design of a large instrument is based on using four collectors of moderate size (12 m)

\subsection{A design for a large stellar intensity interferometer}

To illustrate the potentialities and the limitations of an intensity interferometer let us look briefly at the design ${ }^{3,14}$ which we put forward in March 1971 as a possible successor to the interferometer at Narrabri. The parameters of this proposed design are shown in Table I. 
TABLE I

\section{$\underline{\text { Parameters of a proposed large interferometer }}$}

$\begin{array}{lc}\text { Total area of flux-collectors } & 4 \times 80 \mathrm{~m}^{2} \\ \text { Number of optical channels } & 10 \\ \text { Electrical bandwidth } & 1.000 \mathrm{MHz} \\ \text { Number of correlator channels } & 60 \\ \text { Limiting magnitude (V) } & +7.3 \\ \text { Angular resolution } & \sim 3 \times 10^{-5} \mathrm{arc} " \\ \text { Estimated cost (Dec. 1972) } & \text { \$A } 4 \mathrm{~m}\end{array}$

The layout of this instrument differs from the circular layout at Narrabri. Light from the star (Figure 2) is reflected by the flat coelostats $\left(F_{1}, F_{2}\right)$ on to fixed paraboloidal reflectors $\left(P_{1}, P_{2}\right)$ which focus 1 on to fixed detectors $\left(D_{1}, D_{2}\right)$ The coelostats are mounted on straight railway tracks running east and west. As the star moves across the sky their separation is controlled by a computer so that the projected baseline $A B$ remains constant, and their relative distances from the two fixed reflectors are controlled so that the light from the star arrives at the two detectors at the same time making

$$
\mathrm{AF}_{1}+\mathrm{F}_{1} \mathrm{P}_{1}=\mathrm{BF}_{2}+\mathrm{F}_{2} \mathrm{P}_{2}
$$

This configuration was chosen for two principal reasons. Firstly, unlike a circular track, it can be enlarged without radical modification. Second1y, it avoids the very awkward technical problem of making a multichannel wide-band variable delay for the outputs of the phototubes. The principal disadvantages are that the railway tracks are 1onger than the projected baseline and that the effective area of the coelostats is reduced by their inclination to the optical axis of the reflectors.

In practice the simple configuration shown in Figure 2 would not be satisfactory because, in order to reach a limiting magnitude of +7 , the coelostats and paraboloids would have to be inconveniently large. 

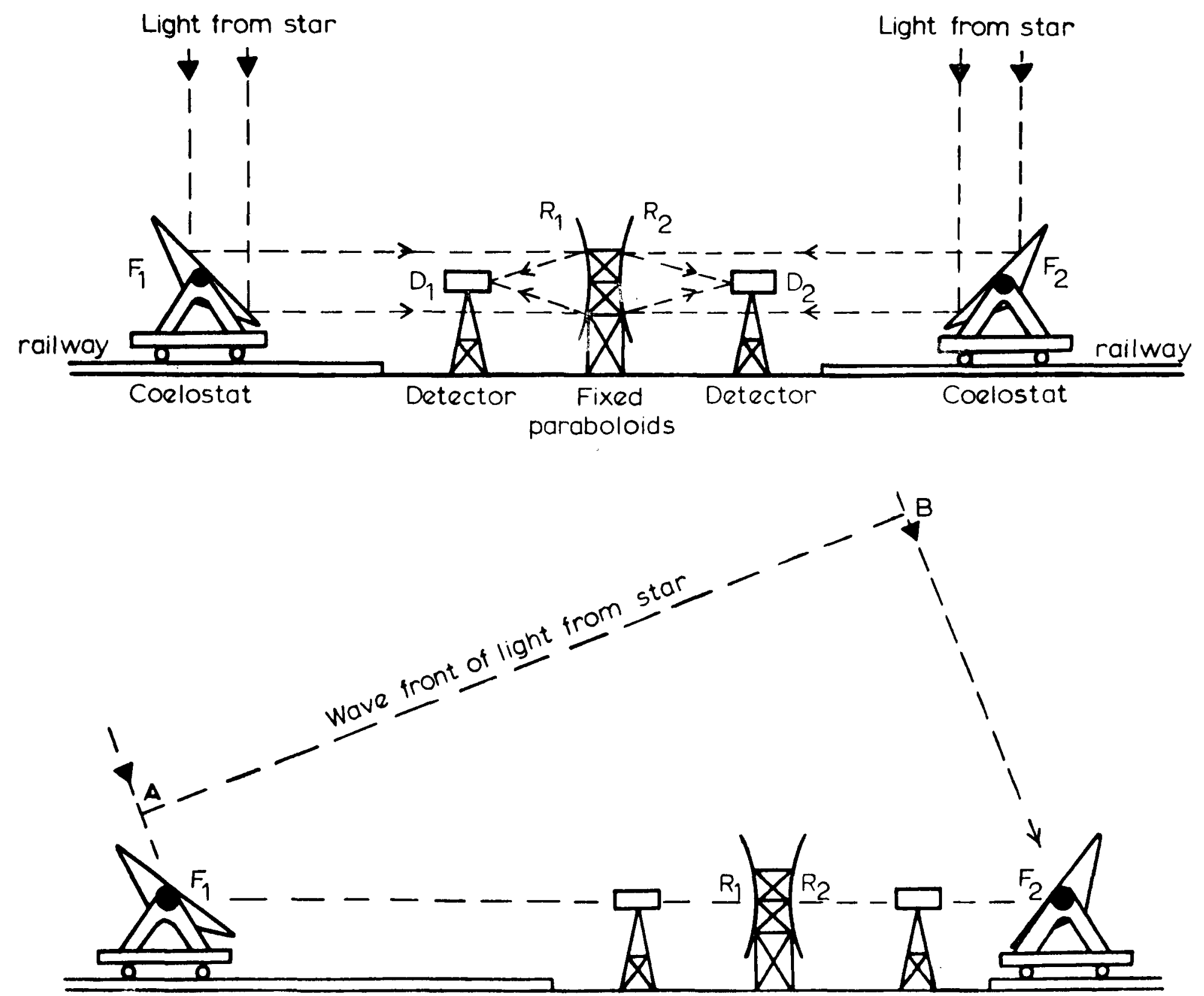

Figure 2. The basic layout of the proposed interferometer when observing a star in the zenith and a star at $70^{\circ}$ elevation due west.

Their physical size would, due to partial resolution, complicate the interpretation of the measurements on some stars; furthermore, it would seriously limit the number of short baselines. The only solution is to use a number of smaller flux-collectors and the optimum design is, of course, a difficult question of cost and complication.

After studying this problem in some detail we chose to divide the instrument into two identical parts using parallel railway tracks; thus there are 4 flats, 4 fixed paraboloids and 4 detectors. The diameter of the flats and paraboloids is $12 \mathrm{~m}$ and the tolerances on their surfaces are such that the field of view of the detectors is 3 mins of arc (on the sky) and this 
performance is maintained at wind speeds up to at least $35 \mathrm{~km} \mathrm{~h}^{-1}$.

The electronic correlator was designed to make füll use of all the available information. There are 4 coelostats, giving 6 possible baselines simultaneously, and 10 separate optical channels. Thus, if measurements are to be made at all these baselines simultaneously, the correlator must have 60 independent channels each with a bandwidth of $1000 \mathrm{MHz}$.

After studying the design and cost of the most "difficult" parts of this proposal (e.g. the coelostats, multi-channel optical filters, wide-band correlator etc.) we can see no serious difficulty in building a very large intensity interferometer. We are confident that it would work well at very long baselines $(\sim 2 \mathrm{~km})$ and in the presence of atmospheric scintillation and would yield reliable measurements on thousands of stars. The cost would be roughly equivalent to that of an 84 inch $(2.3 \mathrm{~m})$ telescope complete with dome and accessories.

A less satisfactory feature of our proposed design is that the limiting magnitude is +73 , whereas we would prefer +9 . For that reason we have looked hard at the problem of increasing the sensitivity. As we have already seen, it is not easy to increase the electrical bandwidth, the number of optical channels or the exposure time. The only remaining options are to increase the total area of the flux-collectors or the quantum efficiency of the photodetectors.

In our present design the quantum efficiency $(\alpha)$ of the photomultipliers was taken as 0.20 (at $443 \mathrm{~nm}$ ) and their bandwidth (bv) as $1000 \mathrm{MHz}$. If the product $\alpha \mathrm{b}_{\mathrm{v}}^{\frac{1}{2}}$ could be improved, without introducing excess noise, then the sensitivity of the interferometer could be increased. At the present time we know of no device which promises a significant improvement. The only remaining possibility is to increase the area of the flux-collectors, but, as we have seen, there are significant objections to increasing their diameter. It would of course be possible to increase the number of flux-collectors, possibly in a different configuration to the one we have chosen, but this cannot be done without increasing the total cost which, in our opinion, is already quite high enough. In short we can see no reasonable way of increasing the limiting magnitude of our proposed instrument much beyond +73 . 


\subsection{Conclusion}

It would be possible, without any serious technical difficulty, to build a large stellar interferometer to measure stars brighter than magnitude $+7(\mathrm{~V})$ with an accuracy better than $\pm 5 \%$. Such an instrument would work reliably at very long ( $\sim 2 \mathrm{~km}$ ) baselines and in the presence of atmospheric scintillation. The instrument would be large and would cost about the same amount as an 84 inch (2.3m) telescope with dome and accessories. With present-day techniques it would be difficult to extend the limiting magnitude much beyond +7 , for example to +9 , without excessive cost and complication.

\section{REFERENCES}

1. R. Hanbury Brown, J Davis and L.R. Allen, Mon. Not. R. astr. Soc., $137,375,1967$.

2. R. Hanbury Brown, J Davis, L.R. Allen and J.M. Rome, Mon. Not. R. astr. Soc., 137, 393, 1967 .

3. R. Hanbury Brown, "The Intensity Interferometer" (Taylor and Francis, London, 1974).

4. R. Hanbury Brown, J. Davis and L.R. Allen, Mon. Not. R. astr Soc., $\underline{167}, 121,1974$.

5. A.D. Code, J. Davis, R.C. Bless and R. Hanbury Brown, Ap. J., 203, 417, 1976.

6. D. Herbison-Evans, R. Hanbury Brown, J. Davis and L.R. Allen, Mon. Not. R. astr. Soc., 151, 161, 1971.

7. R. Hanbury Brown, J. Davis, D. Herbison-Evans and L.R. Allen, Mon. Not. R. astr. Soc., $148,103,1970$.

8. R. Hanbury Brown, J. Davis and L.R. Allen, Mon. Not. R. astr Soc., $\underline{168}, 93,1974$.

9. R. Hanbury Brown, J. Davis, R.J.W. Lake and R.J. Thompson, Mon. Not. R. astr. Soc., 167, 475, 1974 .

10. L.R. Allen and R.H. Frater, Proc. Instn. elect. Engrs., 117, 1603, 1970.

11. R. Hanbury Brown, J. Davis and L.R. Allen, Mon. Not. R. astr. Soc., $146,399,1969$. 
12. R. Hanbury Brown and R.Q. Twiss, Proc. R. Soc. A, 243, 199, 1958.

13. J. Davis, This volume p 1-1.

14. J Davis, "Multicolour Photometry and the Theoretical HR Diagram,

(Ed. A.G.D. Philip and D.S. Hayes, Dudley Observatory Report No.9, $199,1975$.

15. J.W.M. Baars and B.G. Hooghoudt, Astron. and Astrophys., 31, 323, 1974.

16. V I. Tatarskii, "The effects of the turbulent atmosphere on wave propagation", (Israel Programme of Scientific Translations) Jerusalem, 1971.

17. D.L. Fried, J Opt Soc. Am., 55, 1427, 1965. 